\title{
Multiobjective Synthesis of Steerable UWB Circular Antenna Array considering Energy Patterns
}

\author{
Leopoldo A. Garza, ${ }^{1}$ Marco A. Panduro, ${ }^{1}$ David H. Covarrubias, ${ }^{1}$ and Alberto Reyna ${ }^{2}$ \\ ${ }^{1}$ CICESE Research Center, Km 107 Carretera Tijuana-Ensenada, 22860 Ensenada, BCN, Mexico \\ ${ }^{2}$ Universidad Autónoma de Tamaulipas, UAMR-R, Carretera Reynosa-San Fernando, 88779 Reynosa, TAMPS, Mexico \\ Correspondence should be addressed to Leopoldo A. Garza; lgarza@cicese.edu.mx
}

Received 31 July 2015; Accepted 7 October 2015

Academic Editor: Kerim Guney

Copyright (c) 2015 Leopoldo A. Garza et al. This is an open access article distributed under the Creative Commons Attribution License, which permits unrestricted use, distribution, and reproduction in any medium, provided the original work is properly cited.

\begin{abstract}
True-time delay antenna arrays have gained a prominent attention in ultrawideband (UWB) applications such as directional communications and radar. This paper presents the design of steerable UWB circular array by using a multiobjective time-domain synthesis of energy pattern for circular antenna arrays. By this way we avoid individual beamforming for each frequency in UWB spectrum if the problem was addressed from the frequency domain. In order to obtain an energy pattern with low side lobe level and a desired main beam, the synthesis presented is performed by optimizing the true-time delays and amplitude coefficients for the antenna elements in a circular geometry. The method of Differential Evolution for Multiobjective Optimization (DEMO) is used as the optimization algorithm in this work. This design of steerable UWB circular arrays considers the optimization of the truetime exciting delays and the amplitude coefficients across the antenna elements to operate with optimal performance in the whole azimuth plane $\left(360^{\circ}\right)$. A comparative analysis of the performance of the optimized design with the case of conventional progressive delay excitations is achieved. The provided results show a good performance for energy patterns and for their respective power patterns in the UWB spectrum.
\end{abstract}

\section{Introduction}

In recent times, ultrawideband (UWB) communications require antenna arrays for applications such as beamforming and directional communications with high rate data transmission and high sensitivity $[1,2]$. In such applications, the UWB antenna arrays are required to establish a point-topoint communication with suppression of undesired interferences [3]. However, the power pattern changes significantly between the lower and upper edge of the UWB frequency band $(3.1 \mathrm{GHz}$ to $10.6 \mathrm{GHz})$ defined by the US Federal Communications Commission (FCC) [4]. This implies a complex design problem due to numerous radiation patterns if the analysis in the frequency domain is used [5]. An alternative approach is the analysis in the time domain to design UWB antenna arrays. The pattern in the time domain is defined as the energy pattern [6]. The energy pattern of the antenna array is the total response of the power patterns in the frequency domain [7]. Therefore, using the energy pattern in the time domain, we avoid the individual beamforming for the power patterns in the frequency domain.

Time-domain (TD) or pulsed antenna arrays have recently been studied by pondering their advantages of low side lobe levels (SLL) and high resolution for applications such as communications, remote sensing, and radar [8]. The design of TD linear antenna array has been reported in [9-13], exploiting its features of fixed and scanning beam patterns. The synthesis of linear and circular antenna arrays has been reported in $[14,15]$ by modelling HermiteRodriguez waveforms functions to obtain arrays currents and the energy pattern by a generalized hypergeometric function. A TD UWB transverse electromagnetic horn antenna array optimized by a Micro Genetic Algorithm is presented in [16]. Furthermore, a nonuniform UWB linear antenna array optimized by the well-known Differential Evolution Method for low side lobe level during beam-scanning is presented in [17]. 


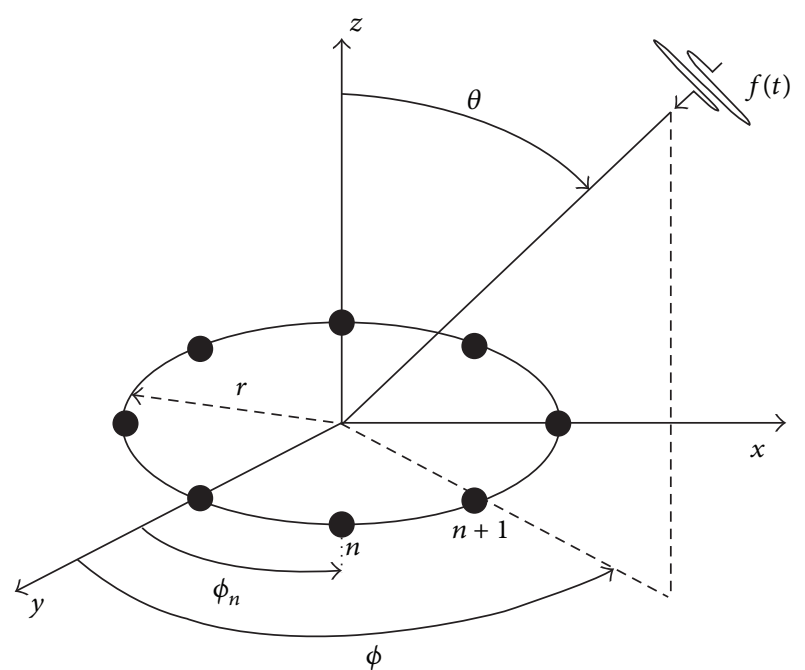

FIgURE 1: Time-domain circular antenna array model.

Moreover, two-dimensional TD arrays with scanning beam pattern by using the Sequential Quadratic Programming to find the optimum positions, amplitudes, and time delays are presented in [18-20].

Now, our proposal presents the synthesis of TD circular antenna arrays for UWB spectrum. Particularly, our research considers the synthesis of a single-ring circular antenna array with low SLL and beamwidth and different requirements for a steerable main lobe. This design of steerable UWB circular arrays considers the optimization of the true-time exciting delays and the amplitude coefficients across the antenna elements to operate with optimal performance in the whole azimuth plane $\left(360^{\circ}\right)$. The performance of UWB circular array could be improved substantially (with respect to the UWB circular array with the conventional progressive delay excitation), if the amplitude and delay excitations are set or optimized in an adequate way. The synthesis process is carried out by Differential Evolution for Multiobjective Optimization (DEMO) [21] by finding out the optimal true-time exciting delays and amplitude coefficients of pulsed antenna elements. Hence, the novelty of this research is the synthesis of TD circular antenna array in order to obtain steerable energy patterns with low SLL; by this way we avoid the individual beamforming which would require an optimization process for each frequency in UWB spectrum.

The remainder of the paper is organized as follows: Section 2 presents the time-domain formulation for a steerable UWB circular antenna array model. Section 3 describes the synthesis algorithm, DEMO, to achieve a steerable energy pattern with low SLL. The optimization results are shown in Section 4. Finally, conclusions are presented in Section 5.

\section{Time-Domain Antenna Array Model}

Let us assume a TD circular antenna array of $N$ antenna elements grouped in a circular geometry located in the plane $X-Y$ as shown in Figure 1. Each antenna is fed by a short pulse with different time delays and amplitude coefficients.
The short pulses are added in space resulting in far-field waveform. Then, the far-field waveform will depend basically on four parameters referred to as the waveform of feeding short pulse, the antenna element positions, the time delays among the short pulses, and the amplitude coefficients [1].

It is known that the 5th derivative Gaussian pulse has the most effective pulse shape with power spectrum under FCC limitation floor, and this pulse can be transmitted without additional filtering [22]. For this reason, let us consider the 5 th derivative Gaussian pulse as the waveform of the receiving short pulses to each antenna element as follows $[22,23]$ :

$$
\begin{aligned}
& f_{n}(t) \\
& =A\left(-\frac{t^{5}}{\sqrt{2 \pi} \sigma^{11}}+\frac{10 t^{3}}{\sqrt{2 \pi} \sigma^{9}}-\frac{15 t}{\sqrt{2 \pi} \sigma^{7}}\right) \exp \left(-\frac{t^{2}}{2 \sigma^{2}}\right),
\end{aligned}
$$

where $A$ is the amplitude of the pulse and $\sigma$ is a time constant. In order to satisfy the FCC regulations, these parameters are chosen to be $A=1, \sigma=51 \mathrm{ps}$, and $t=250 \mathrm{ps}$ [22-24]. Now, the radiation properties of the TD circular antenna array can be modelled. Then, by using the pattern descriptors developed by [25], the energy pattern can be defined as

$$
P_{\text {energy }}(\theta, \phi)=\int_{0}^{t}|s(t, \theta, \phi)|^{2} d t,
$$

where $s(t, \theta, \phi)$ is the far-field waveform of the antenna array and the direction $(\theta, \phi)$ represents, respectively, the elevation and azimuth angles. The spectrum in frequency of $s(t, \theta, \phi)$ or power patterns can be obtained by a Fourier transform as follows [25]:

$$
P(f, \theta, \phi)=\left|\int_{-\infty}^{\infty} s(t, \theta, \phi) e^{-j 2 \pi f t} d t\right|,
$$

where $f$ is the frequency from $3.1 \mathrm{GHz}$ to $10.6 \mathrm{GHz}$ defined by the FCC regulations for UWB systems [22-24].

Then, the far-field waveform for the TD circular antenna array shown in Figure 1 is defined as follows [26]:

$$
s(t, \theta, \phi)=\sum_{n=1}^{N} a_{n} f_{n}\left(t-\Delta t+\tau_{p}-\tau_{n}, \theta, \phi\right),
$$

where

$$
\begin{aligned}
\Delta t & =\frac{r}{c} \sin \theta \cos \left(\phi_{n}-\phi\right), \\
r & =\sum_{n=1}^{N} \frac{d_{n}}{2 \pi}, \\
\phi_{n} & =\sum_{n=1}^{N} \frac{2 \pi n}{N}, \\
\tau_{p} & =\frac{r}{c} \sin \theta_{0} \cos \left(\phi_{n}-\phi_{0}\right) .
\end{aligned}
$$

The variable $N$ is the number of antenna elements in the array; $a_{n}$ is the amplitude coefficient of the antenna element $n, d_{n}$ is the Euclidian space between antenna elements, $r$ is 
the radius of the circle array, and $c$ is the propagation speed. The direction $\left(\theta_{0}, \phi_{0}\right)$ is the desired maximum direction. The term $\phi_{n}$ is the angular position of the element $n ; \tau_{p}$ is the progressive exciting time delay; and $\tau_{n} \in\left[0, \tau_{\max }\right]$ is the disturbed exciting time delay.

The proposed synthesis problem is to obtain an optimum energy pattern by optimizing the amplitude excitations and the disturbing exciting time delay of a circular antenna array for a maximum performance in terms of the side lobe level and a desired main beamwidth in a scanning range of $\left[-180^{\circ}, 180^{\circ}\right]$. The design of a steerable UWB circular antenna array could be achieved by considering the rotation properties of monochromatic circular arrays; that is, the rotation properties of circular arrays (for just one frequency) could be extended to design steerable UWB circular arrays considering energy patterns.

This design rule is very simple. Let us consider a circular array of $N$ elements to illustrate this point. In this case, the behavior of the array factor for the scanning range of $-180 \leq \phi_{n} \leq 180^{\circ}$ is considered (with $\phi_{0}$ as the beam direction in azimuth plane). If the number of antennas is $N=8$ and there is uniform angular separation of $45^{\circ}$ between different beam steering angles, the optimal excitation solution (amplitudes and time delays) for a particular beam steering angle should also apply to other beam steering angles by substituting $\left(a_{n}, \tau_{n}\right)$ into $\left(a_{n+1}, \tau_{n+1}\right)$ when the beam steering is increased $\left(\phi_{0}+45^{\circ}\right)[27,28]$.

Through our proposal, only one optimization is required for beam steering in steps of $45^{\circ}$ (in all azimuth plane) instead of one optimization for each beam steering direction. Then, the objective functions for this design case, of ${ }_{1}$ and of $f_{2}$, set to be minimized are defined as follows:

$$
\begin{aligned}
& \mathrm{of}_{1}(n)=P_{\text {energy }}\left(\theta_{\mathrm{SLL}}, \phi_{\mathrm{SLL}}\right), \\
& \mathrm{of}_{2}(n)=\mathrm{BW}\left(P_{\text {energy }}\right) .
\end{aligned}
$$

The proposed of ${ }_{1}$ regards the maximum peak side lobe level represented by term $P_{\text {energy }}\left(\theta_{\text {SLL }}, \phi_{\text {SLL }}\right)$, and proposed of ${ }_{2}$ regards the beamwidth of the obtained energy pattern and it is represented by term $\mathrm{BW}\left(P_{\text {energy }}\right)$.

The present study will be focused on the behavior of the pattern descriptor (using the energy pattern in time domain) generated by the UWB circular antenna array. In particular, this design problem is not focused on any type of antenna element. In this case, the design problem is set at the array structure level, that is, the best design configuration (amplitude and time delays excitations), when a circular array structure with a steerable pattern is employed in UWB systems.

Then, in order to solve this design problem, the method of Differential Evolution for Multiobjective Optimization (DEMO) is used in order to find the spread of solutions and the convergence to an optimum solution to minimize both objective functions. The procedure of the optimization process is described in the next subsection.

\section{Differential Evolution for Multiobjective Optimization}

The optimization process is carried out by the algorithm of DEMO for the effectiveness in antenna arrays design [29]. DEMO is an optimization approach based on the classical method of Differential Evolution (DE) [30] combined with the mechanisms of Pareto-based ranking and crowding distance sorting, employed in the literature of evolutionary algorithms for multiobjective optimization.

In order to resolve the proposed synthesis design problem, the DEMO algorithm was selected based on the performance comparison of NSGA-II [31], EM-MOPSO [32], and DEMO variants [21] made by Panduro et al. [29] for the design of circular concentric rings antenna arrays. The results of that performance comparison showed that the variants of DEMO/parent and DEMO/closest/dec found better nondominated solutions as an approximation to the Pareto solution. Moreover, the performance comparison also evaluates the metric of the binary epsilon indicator [33] to calculate how much the nondominated front of each optimization algorithm is separated from the Pareto front. DEMO/parent outperforms totally the other algorithms by having a better behavior on this metric and a better approximation to the Pareto front. Based on these comparisons for the design of antenna arrays over a circular geometry, DEMO/parent is implemented in this synthesis of steerable UWB circular antenna arrays.

The procedure for used DEMO/parent is described as follows [21]:

(1) Evaluate the initial population $\mathscr{P}$ of random individuals.

(2) While stopping criterion not met, do the following:

(a) For each individual $P_{i}(i=1, \ldots$, popSize $)$ from $\mathscr{P}$ repeat:

(i) Create candidate $C$ from $P_{i}$.

(ii) Evaluate candidate.

(iii) If the candidate dominates the parent, the candidate replaces the parent.

If the parent dominates the candidate, the candidate is discarded.

Otherwise, the candidate is added in the population.

(b) If the population has more than popSize individuals, truncate it sorting the individuals with nondominated sorting and then evaluating the individuals of the same front with the crowding distance metric.

(c) Randomly enumerate the individuals in $\mathscr{P}$.

For the candidate creation, the DE scheme DE/rand/1/bin $[21,30]$ is used, and the procedure for this scheme is described as follows:

(1) Randomly select three individuals $P_{i_{1}}, P_{i_{2}}$, and $P_{i_{3}}$ from $\mathscr{P}$, where $i, i_{1}, i_{2}$, and $i_{3}$ are pairwise different. 


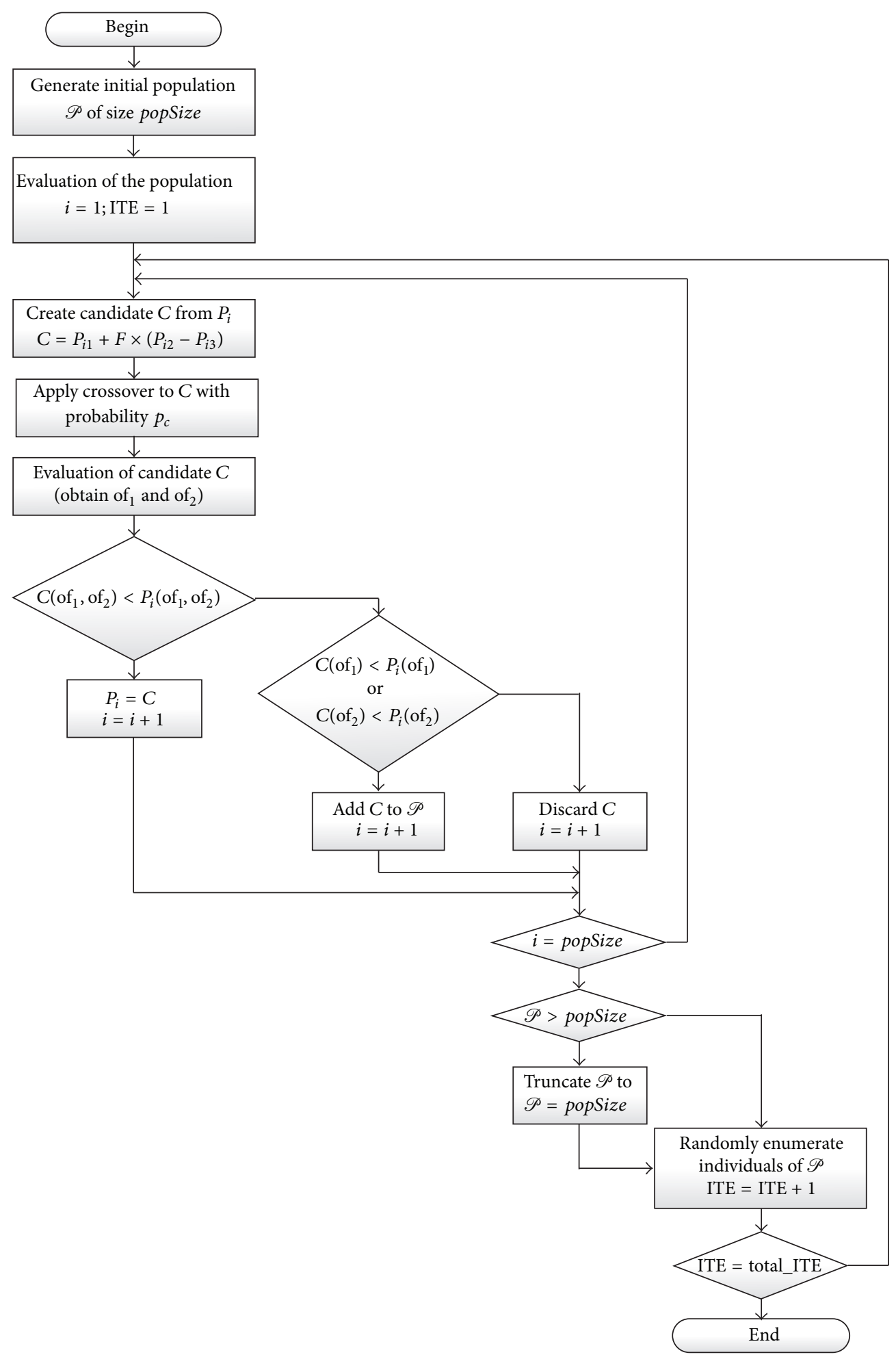

FIGURE 2: Flow chart for the implemented DEMO/parent procedure.

(2) Calculate candidate $C$ as $C=P_{i_{1}}+F \times\left(P_{i_{2}}-P_{i_{3}}\right)$, where $F$ is a scaling factor.

(3) Modify the candidate by binary crossover with the parent using crossover probability $p_{c}$.
The immediate replacement of the parent individual with the candidate that dominates it emphasizes elitism within reproduction, helping achieve the convergence to the true Pareto front. And the use of nondominated sorting and crowding distance metric in truncation of the extended 
population stimulates the uniform spread of solutions, finding as many nondominated solutions as possible [21].

Therefore, the procedure of the $\mathrm{DEMO} /$ parent process for our synthesis problem is described in Figure 2. The individuals are encoded in a vector of real numbers that represents the amplitudes excitations on the range $\left(0, a_{\max }\right)$, and a vector of real numbers restrained on the range $\left(0, \tau_{\max }\right)$ that represents the disturbing exciting time delay. And the stopping criterion to meet is the total number of iterations.

In the next section, the results of using this optimization process in the synthesis of TD circular antenna arrays are presented.

\section{Simulation Results}

The method DEMO/parent was implemented to optimize the disturbed exciting time delays and amplitude excitations of TD circular antenna array. For the case of a steerable energy pattern, the main beam is steered in the azimuth plane $-180^{\circ} \leq \phi_{0} \leq 180^{\circ}$ with an angular step of $45^{\circ}$ in the cut of $\theta=\theta_{0}=90^{\circ}$. Since the principal advantage of the circular array is steering the beam without significant changes over the pattern, we evaluate the rotation properties in all azimuth plane, but this property could be extended to consider the elevation plane. Therefore, the number of elements for the circular antenna array is given by $N=360^{\circ} / 45^{\circ}=8$, with Euclidean spacing of $150 \mathrm{~mm}$ among the antenna elements. According to [10] this spacing value can be enough to avoid important coupling effects. For generation of individuals, the maximum value of amplitudes excitation $a_{\max }$ was set to 10 , and the maximum value of disturbing exciting time delay $\tau_{\max }$ we have considered is $100 \mathrm{ps}$. For the parameters of the $\mathrm{DEMO} /$ parent algorithm, we have set the population size to 600. We consider a scaling factor $F=0.5$ and a two-point crossover with a crossover probability of $p_{c}=0.15$. And the stopping criterion is 600 iterations.

The algorithm was executed 10 times and the consolidated front for each run is considered as the nondominated front. Figure 3 shows the nondominated front of both objective functions obtained by the $\mathrm{DEMO} /$ parent algorithm for the circular antenna array proposed. The result shown in Figure 3 illustrates that the DEMO/parent algorithm found nondominated solutions for the two objective functions. Furthermore, this figure shows the selected design solution from the nondominated front. As shown in Figure 3, this design solution represents the best compromise between the side lobe level and beamwidth for a steerable energy pattern. As additional information, the nondominated front was obtained after the 600 iterations in an average time of 13415 seconds, for a population of 600 .

Figure 4 illustrates the selected solution from the nondominated front for the steerable energy patterns considering the circular antenna array with amplitude excitations and disturbing exciting time delay optimized by $\mathrm{DEMO} /$ parent algorithm. The sets of amplitudes and time delays provide the normalized energy pattern with a maximum SLL $\leq$ $-12.7014 \mathrm{~dB}$ and a maximum $\mathrm{BW} \leq 11.52^{\circ}$ for the beam steering directions of (a) $\phi_{0}=-135^{\circ}$, (b) $\phi_{0}=-90^{\circ}$, (c) $\phi_{0}=$

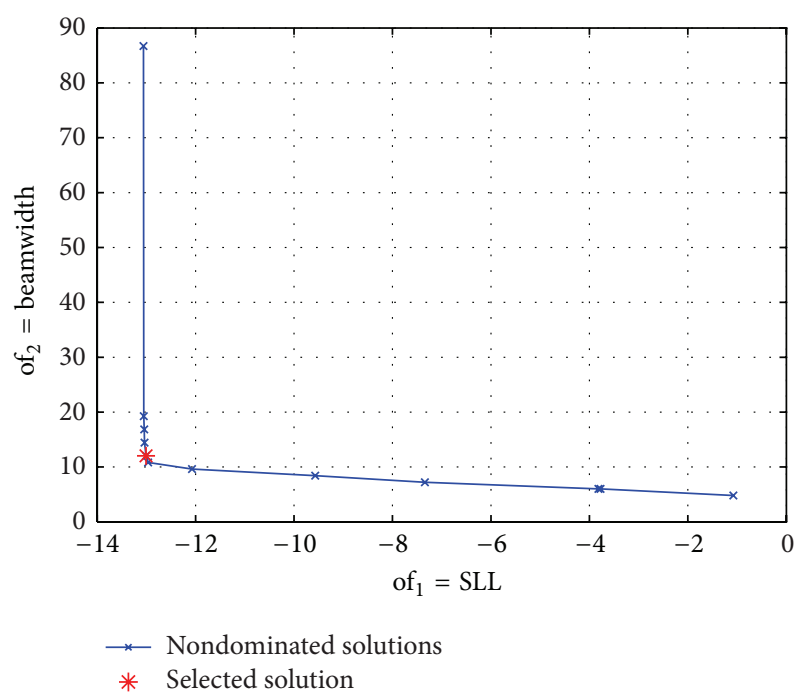

FIGURE 3: Nondominated front obtained by DEMO/parent algorithm and the selected solution for steerable energy pattern of a circular antenna array.

$-45^{\circ}$, (d) $\phi_{0}=0^{\circ}$, (e) $\phi_{0}=45^{\circ}$, (f) $\phi_{0}=90^{\circ}$, (g) $\phi_{0}=135^{\circ}$, and (h) $\phi_{0}=180^{\circ}$. Each energy pattern is compared against a conventional case of circular antenna array with uniform amplitude excitations and progressive delay excitation. From Figure 4, we can conclude that the DEMO algorithm obtained a reduction of the SLL and kept a beamwidth narrow for all beam steering directions. It is essential to mention that the obtained values of SLL and beamwidth for the optimized energy pattern are the same when the main beam is steered in the proposed directions, by rotating the optimized set of amplitude excitations and the disturbing exciting time delays in the circular antenna array.

The numerical values of the side lobe level and beamwidth for the optimized steerable energy pattern and its sets of amplitudes and time delays are presented in Table 1.

It is observed in Table 1 that the DEMO algorithm could minimize the SLL and maintain a narrow beamwidth with respect to the conventional case. Moreover, this table shows the amplitude excitations and disturbing exciting time delays required to generate the energy pattern for the direction of $\phi_{0}=0^{\circ}$. Please note that, to generate the energy pattern in other directions, the rotation properties (mentioned previously for the circular array proposed) are applied.

Now, in order to observe the effect of the time-domain synthesis over the response in the frequency domain, we calculated the spectrum in frequency of the far-field waveform in time domain by using (3). Figure 5 shows the spectrum in frequency for all UWB frequency band of (a) optimized solution and (b) conventional case, for $\phi_{0}=0$. Because there are no variations when the main beam is steered to the proposed directions, one case is enough to observe the effect of the time-domain synthesis over the response in the frequency domain.

Figure 5 illustrates that the different power patterns show variations in the SLL, with a maximum SLL of $-8.5134 \mathrm{~dB}$ 


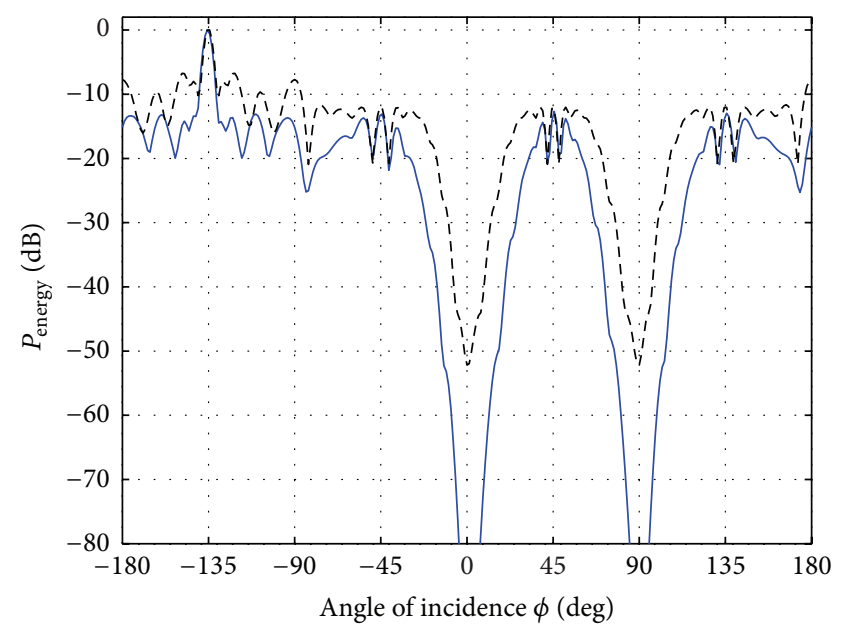

- Optimization solution

- - - Progressive delay

(a) $\phi_{0}=-135^{\circ}$

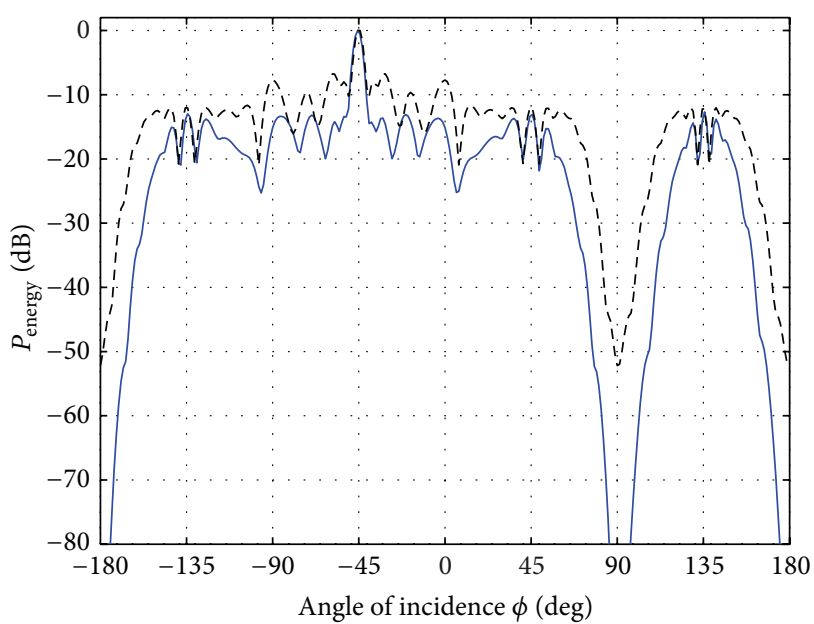

- Optimization solution

- - - Progressive delay

(c) $\phi_{0}=-45^{\circ}$

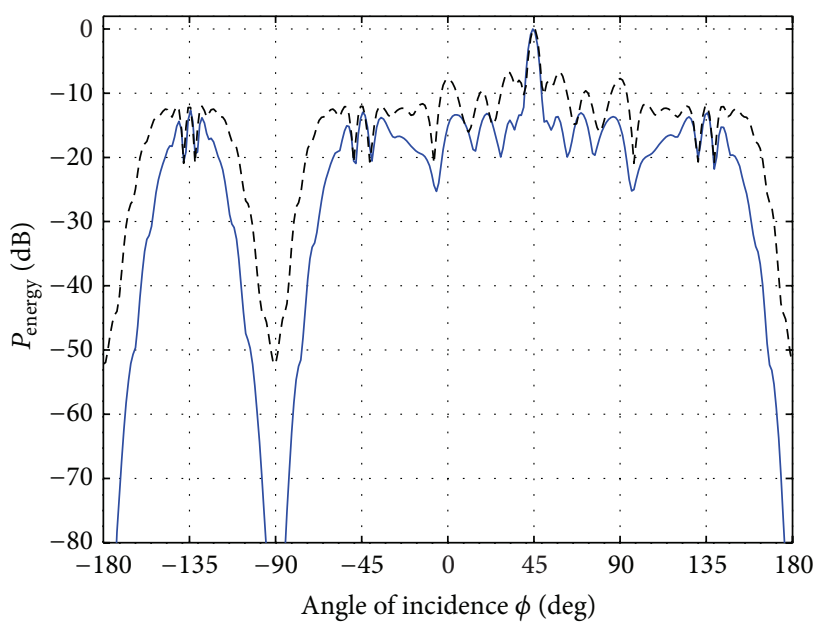

Optimization solution

- - Progressive delay

(e) $\phi_{0}=45^{\circ}$

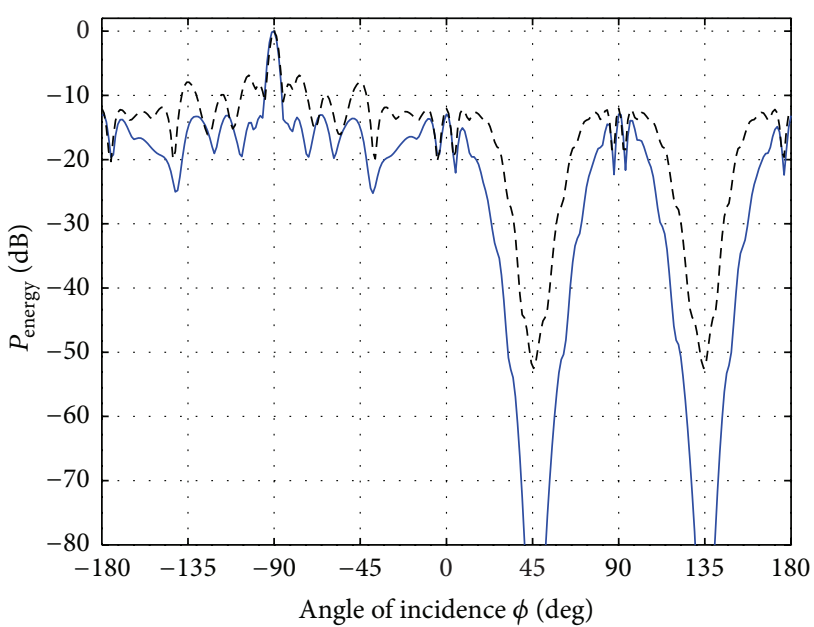

- Optimization solution

- - - Progressive delay

(b) $\phi_{0}=-90^{\circ}$

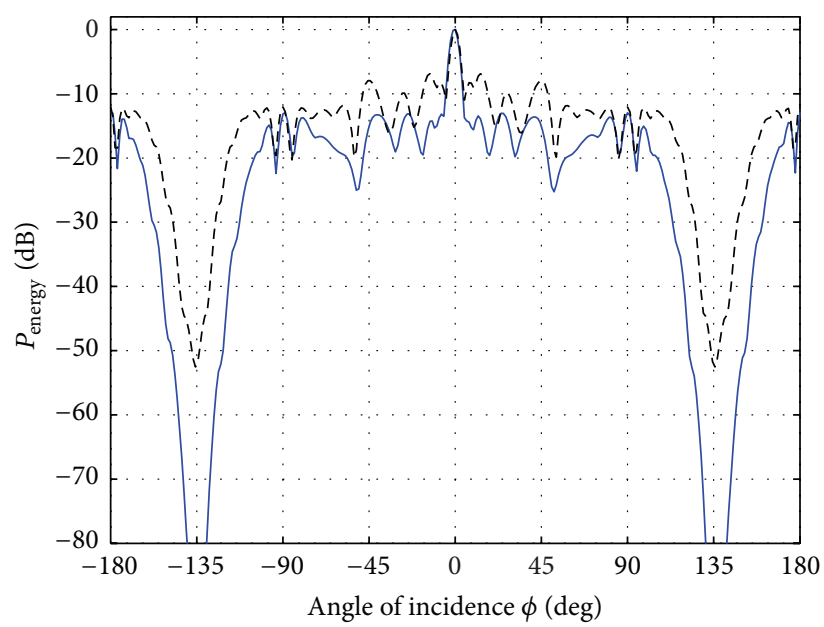

_- Optimization solution

- - - Progressive delay

(d) $\phi_{0}=0^{\circ}$

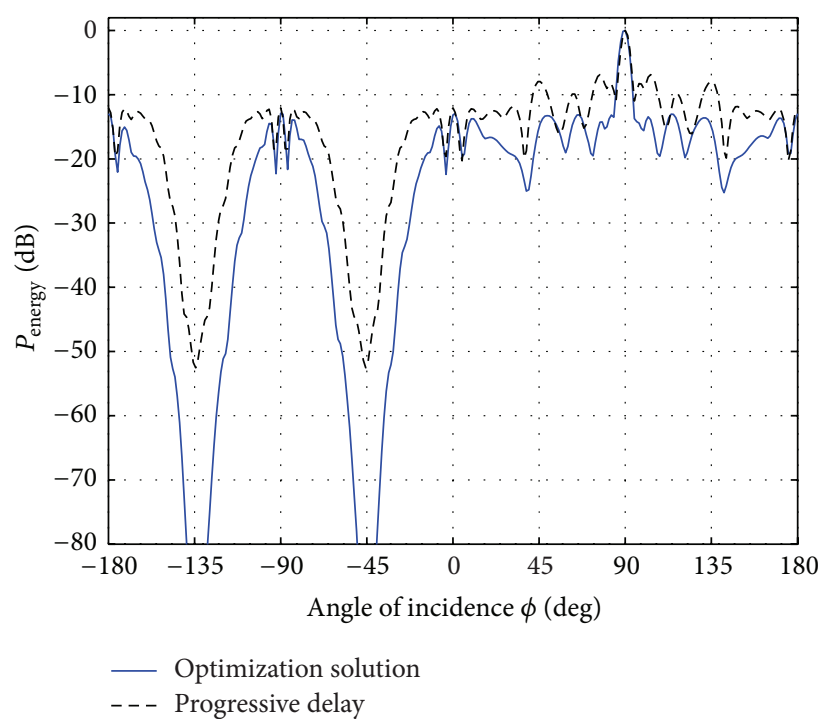

(f) $\phi_{0}=90^{\circ}$

Figure 4: Continued. 


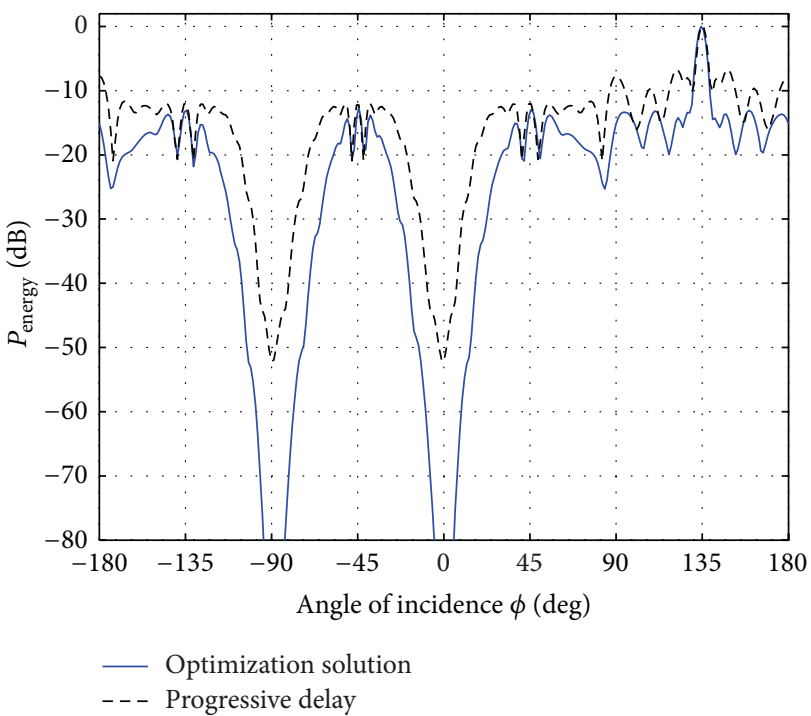

(g) $\phi_{0}=135^{\circ}$

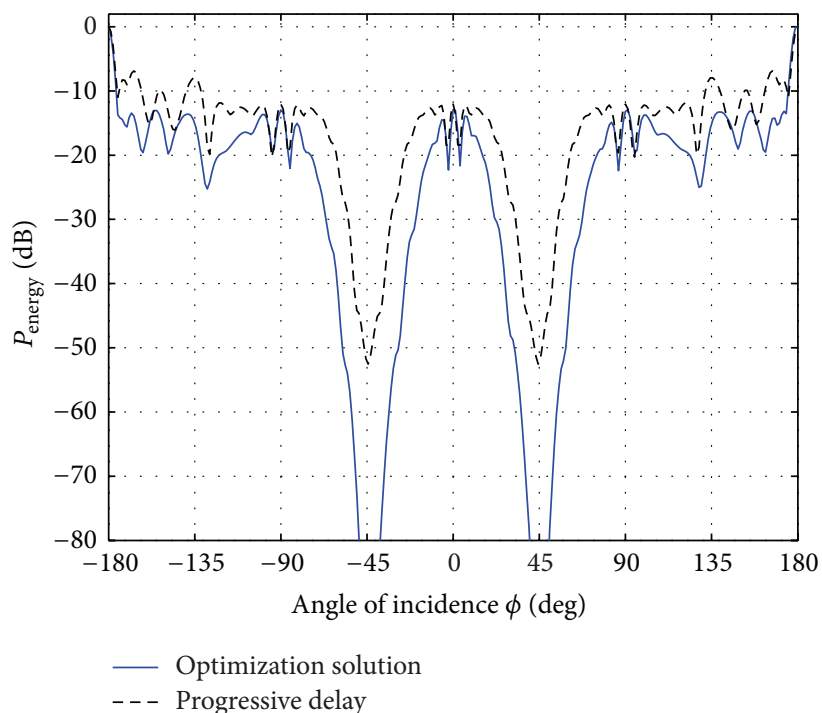

(h) $\phi_{0}=180^{\circ}$

FIGURE 4: Comparison of the energy patterns between conventional case and optimized solution by DEMO/parent algorithm for $(\mathrm{a}) \phi_{0}=$ $-135^{\circ}$, (b) $\phi_{0}=-90^{\circ}$, (c) $\phi_{0}=-45^{\circ}$, (d) $\phi_{0}=0^{\circ}$, (e) $\phi_{0}=45^{\circ}$, (f) $\phi_{0}=90^{\circ}$, (g) $\phi_{0}=135^{\circ}$, and (h) $\phi_{0}=180^{\circ}$.

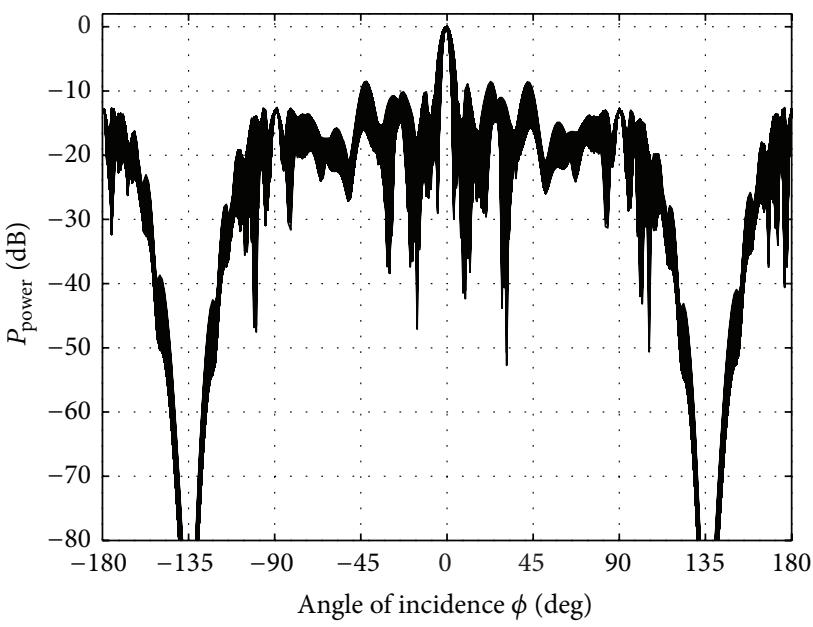

(a) Optimized solution

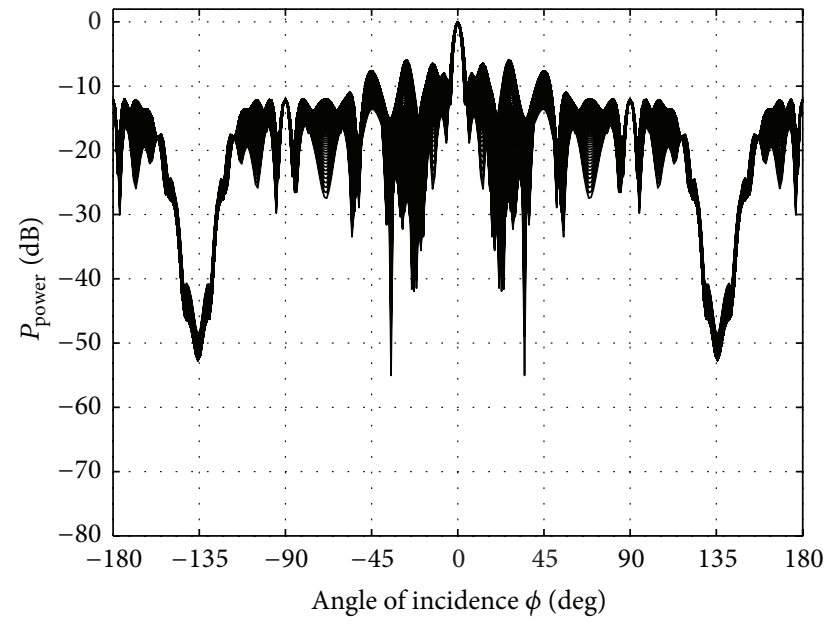

(b) Conventional case

FIGURE 5: Spectrum in frequency of (a) optimized solution and (b) conventional case, for $\phi_{0}=0$.

TABLE 1: Comparison of numerical values between the conventional case and the optimized solution by DEMO/parent for the steerable energy pattern shown in Figure 4 (amplitude and time delays values are for the case $\phi_{0}=0^{\circ}$ ).

\begin{tabular}{|c|c|c|c|c|}
\hline Design case & $\operatorname{Max}$ SLL (dB) & BW (degrees) & Amplitude excitations & Time delays (ps) \\
\hline \multirow{4}{*}{ Conventional case } & \multirow{4}{*}{-6.8731} & \multirow{4}{*}{9.6} & 11 & 00 \\
\hline & & & 11 & 00 \\
\hline & & & 11 & 00 \\
\hline & & & 11 & $0 \quad 0$ \\
\hline \multirow{4}{*}{ Optimized solution } & \multirow{4}{*}{-12.7014} & \multirow{4}{*}{11.52} & $7.1734 \quad 4.9271$ & $77.1268 \quad 87.0812$ \\
\hline & & & $4.1092 \quad 8.5237$ & $84.9614 \quad 87.8917$ \\
\hline & & & $3.8870 \quad 6.4498$ & 72.606975 .0366 \\
\hline & & & $6.9596 \quad 9.0309$ & $73.3821 \quad 69.7864$ \\
\hline
\end{tabular}




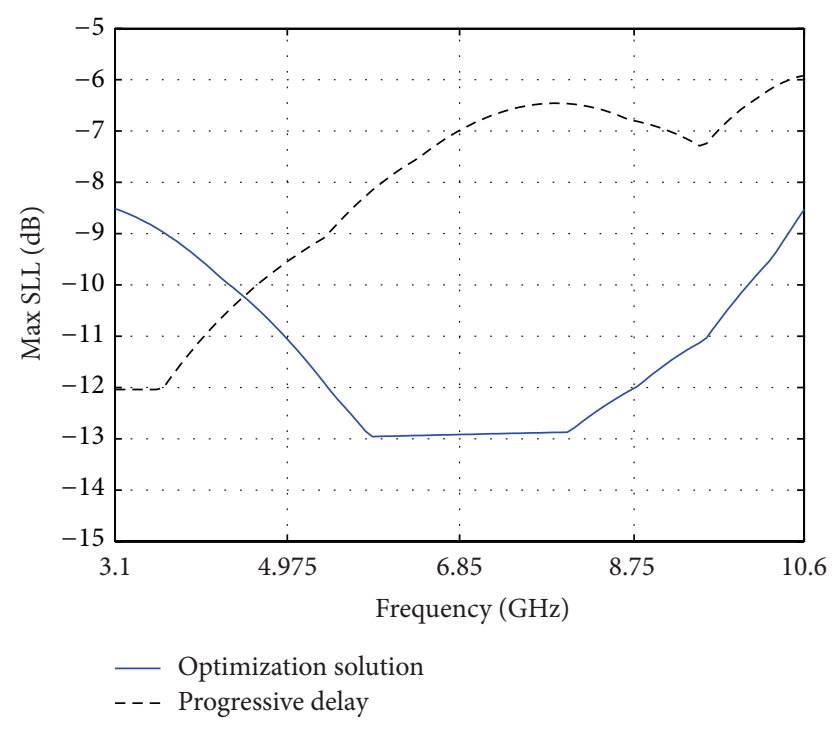

FIGURE 6: Comparison of the maximum SLL of the power pattern in each frequency in the UWB spectrum for the optimized solution and the conventional case.

for the optimized solution and $-5.9157 \mathrm{~dB}$ for the conventional case; and the maximum beamwidth for the optimized solution was $15.6^{\circ}$ and $12^{\circ}$ for the conventional case. These variations are due to many frequencies in the UWB frequency band. Figure 6 presents the variations of the maximum SLL in the spectrum frequency of each case, as it can be observed that the optimization solution has lower SLL over the most part of the UWB frequency band. The variations of SLL are due to wavelength changes in each frequency, and the uniform spacing of the antenna elements in the array caused an increase in the SLL, especially near the boundaries of the spectrum frequency for the optimized solution. Furthermore Figure 7 illustrates the variations of the beamwidth along the frequency spectrum for each case; it is worth mentioning that the value of beamwidth decreases at higher frequency, with a difference (between its boundaries) of $7.2^{\circ}$ for the optimized solution and $2.6^{\circ}$ for the conventional case. This difference is due to the nonuniform set of amplitude excitations and disturbing exciting time delays of the optimized solution.

\section{Conclusions}

This paper presented the synthesis of energy patterns for a TD circular antenna array by using the $\mathrm{DEMO} /$ parent algorithm to optimize the amplitude excitations and the disturbing exciting time delays. The study presented the synthesis process in the time domain for a steerable energy pattern and its effect in the UWB spectrum frequency. The results show a good performance in both proposed cases. For the case of the steerable energy pattern low values of SLL and beamwidth were achieved. The optimized solution presents a reduction of SLL of $5.82 \mathrm{~dB}$ with respect to the conventional case. All patterns in the UWB spectrum resulted in a good performance of SLL and beamwidth. Furthermore, the presented synthesis in time domain is an alternative

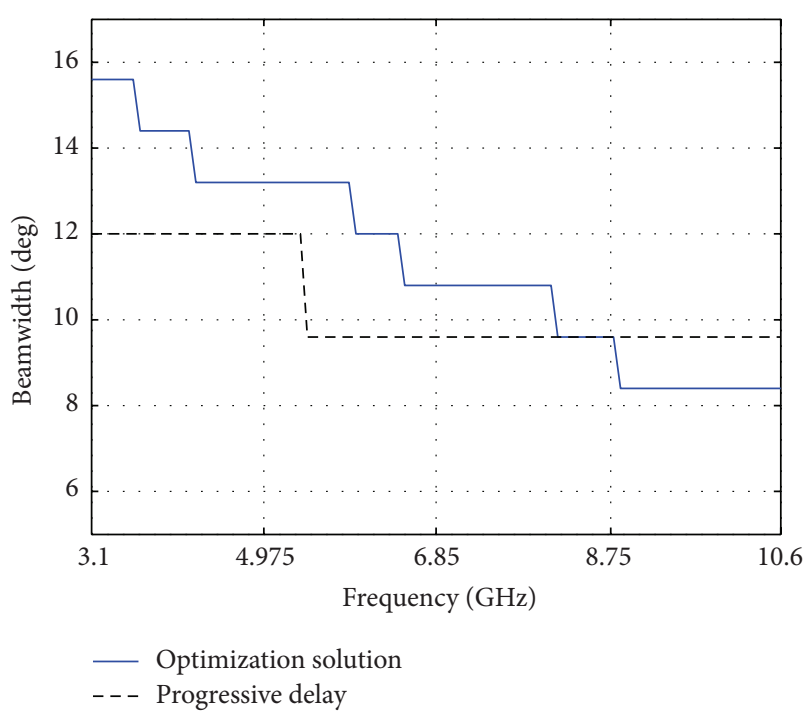

FIgURE 7: Comparison of the beamwidth of the power pattern in each frequency in the UWB spectrum for the optimized solution and the conventional case.

to avoid individual beamforming for each frequency in the UWB spectrum. The proposed time-domain synthesis for the design of circular antenna array along with the application of the rotation properties allowed optimized beam pattern for different steering direction and for the whole UWB spectrum by just one optimization process. Future works can be addressed to include mutual coupling effects of different antenna elements for UWB antenna arrays.

\section{Conflict of Interests}

The authors declare that there is no conflict of interests regarding the publication of this paper.

\section{Acknowledgment}

The research work presented in this paper has been supported by the Mexican Council for Science and Technology (CONACyT), under Grant no. 241163.

\section{References}

[1] T. W. Barrett, "History of ultra wideband communications and radar: part I, UWB communications," Microwave Journal, vol. 44, no. 1, pp. 22-56, 2001.

[2] A. Shlivinski, "Antenna characterization in the time domain," IEEE Transactions on Antennas and Propagation, vol. 45, no. 7, pp. 1140-1149, 1997.

[3] G. Adamiuk, T. Zwick, and W. Wiesbeck, "UWB antennas for communication systems," Proceedings of the IEEE, vol. 100, no. 7, pp. 2308-2321, 2012.

[4] U.S. Federal Communications Commission (FCC), "Revision of part 15 of the commission's rules regarding ultra wideband transmission systems, first report and order," ET Docket 98-153, FCC 02-48, FCC, 2002. 
[5] V. Sipal, B. Allen, D. Edwards, and B. Honary, "Twenty years of ultrawideband: opportunities and challenges," IET Communications, vol. 6, no. 10, pp. 1147-1162, 2012.

[6] S. Werner, C. Sturm, and W. Wiesbeck, "Impulse response of linear UWB antenna arrays and the application to beam steering," in Proceedings of the IEEE International Conference on Ultra-Wideband (ICUWB '05), pp. 275-280, September 2005.

[7] C.-H. Liao, P. Hsu, and D.-C. Chang, "Energy patterns of UWB antenna arrays with scan capability," IEEE Transactions on Antennas and Propagation, vol. 59, no. 4, pp. 1140-1147, 2011.

[8] H. D. Griffiths and A. L. Cullen, "Sidelobe response of antennas to short pulse signals," in Proceedings of the IEEE Radar Conference, pp. 85-90, IEEE, May 2003.

[9] X. H. Wu, A. A. Kishk, and Z. N. Chen, "A linear antenna array for UWB applications," in Proceedings of the IEEE Antennas and Propagation Society International Symposium and USNC/URSI Meeting, pp. 594-597, Washington, DC, USA, July 2005.

[10] S. Chamaani, S. A. Mirtaheri, and M. S. Abrishamian, "Timedomain design of UWB vivaldi antenna array using multiobjective particle swarm optimization," in Proceedings of the 18th Iranian Conference on Electrical Engineering (ICEE '10), pp. 119-123, IEEE, Isfahan, Iran, May 2010.

[11] X.-L. Yuan, G.-F. Zhang, J.-J. Huang, and N.-C. Yuan, "Study of UWB time-domain antenna array scan," in Proceedings of the International Conference on Microwave and Millimeter Wave Technology (ICMMT '08), pp. 1134-1136, Nanjing, China, April 2008.

[12] A. Shlivinski, I. E. Lager, and E. Heyman, "Non-uniform array antennas-the time-domain perspective," in Proceedings of the 7th European Conference on Antennas and Propagation (EuCAP '13), pp. 133-137, Gothenburg, Sweden, April 2013.

[13] D. A. Leatherwood, L. E. Corey, R. B. Cotton, and B. S. Mitchell, "Time-domain properties of phased array antennas," in Proceedings of the IEEE International Conference on Phased Array Systems and Technology, pp. 25-28, May 2000.

[14] M. Ciattaglia and G. Marrocco, "Time domain synthesis of pulsed arrays," IEEE Transactions on Antennas and Propagation, vol. 56, no. 7, pp. 1928-1938, 2008.

[15] G. Marrocco and G. Galletta, "Hermite-rodriguez UWB circular arrays," IEEE Transactions on Antennas and Propagation, vol. 58, no. 2, pp. 381-390, 2010.

[16] Y. Qin, C. Liao, and T. Wei, "Application of micro genetic algorithm to optimization of time-domain ultra-wide band antenna array," in Proceedings of the International Conference on Microwave and Millimeter Wave Technology (ICMMT '07), pp. 1-4, Builin, China, April 2007.

[17] M. A. Panduro and H. Foltz, "Energy Patterns of UWB antenna arrays with low side lobe level during beam-scanning," in Proceedings of the IEEE Antennas and Propagation Society International Symposium (APSURSI'13), pp. 17-18, Orlando, Fla, USA, July 2013.

[18] A. Reyna, M. A. Panduro, G. Romero, J. Villanueva, F. F. Rivera, and A. L. Méndez, "Two-dimensional time-domain antenna arrays for optimum steerable energy pattern with low side lobes," International Journal of Antennas and Propagation, vol. 2014, Article ID 869192, 12 pages, 2014.

[19] A. R. Maldonado, M. A. Panduro, and C. Del-Rio Bocio, "Twodimensional time-domain antenna arrays for steerable energy pattern with low side lobe level," in Proceedings of the 8th European Conference on Antennas and Propagation (EuCAP '14), pp. 1513-1517, IEEE, The Hague, The Netherlands, April 2014.
[20] A. Reyna and M. A. Panduro, "Unequally pulsed arrays for high resolution scanning and low side lobe level," in Proceedings of the IEEE Antennas and Propagation Society International Symposium (APSURSI '14), pp. 832-833, IEEE, Memphis, Tenn, USA, July 2014.

[21] T. Robic and B. Filipic, "DEMO: differential evolution for multiobjective optimization," in Evolutionary Multi-Criterion Optimization, vol. 3410 of Lecture Notes in Computer Science, pp. 520-533, Springer, 2005.

[22] H. Sheng, P. Orlik, A. M. Haimovich, L. J. Cimini Jr., and J. Zhang, "On the spectral and power requirements for ultrawideband transmission," in Proceedings of the International Conference on Communications (ICC '03), pp. 738-742, Anchorage, Alaska, USA, May 2003.

[23] H. Kim and Y. Joo, "Fifth-derivative Gaussian pulse generator for UWB system," in Proceedings of the IEEE Radio Frequency Integrated Circuits Symposium. Digest of Papers (RFIC '05), pp. 671-674, Long Beach, Calif, USA, June 2005.

[24] Z. N. Chen, X. H. Wu, H. F. Li, N. Yang, and M. Y. W. Chia, "Considerations for source pulses and antennas in UWB radio systems," IEEE Transactions on Antennas and Propagation, vol. 52, no. 7, pp. 1739-1748, 2004.

[25] J. S. McLean, H. Foltz, and R. Sutton, "Pattern descriptors for UWB antennas," IEEE Transactions on Antennas and Propagation, vol. 53, no. 1, pp. 553-559, 2005.

[26] S. Foo and S. Kashyap, "Time-domain array factor for UWB antenna array," Electronics Letters, vol. 39, no. 18, pp. 1304-1305, 2003.

[27] M. A. Panduro, C. A. Brizuela, L. I. Balderas, and D. A. Acosta, "A comparison of genetic algorithms, particle swarm optimization and the differential evolution method for the design of scannable circular antenna arrays," Progress In Electromagnetics Research B, no. 13, pp. 171-186, 2009.

[28] A. Reyna, M. A. Panduro, D. H. Covarrubias, and A. Mendez, "Design of steerable concentric rings array for low side lobe level," Scientia Iranica, vol. 19, no. 3, pp. 727-732, 2012.

[29] M. A. Panduro, C. A. Brizuela, J. Garza, S. Hinojosa, and A. Reyna, "A comparison of NSGA-II, DEMO, and EM-MOPSO for the multi-objective design of concentric rings antenna arrays," Journal of Electromagnetic Waves and Applications, vol. 27, no. 9, pp. 1100-1113, 2013.

[30] R. Storn and K. Price, "Differential evolution-a simple and efficient heuristic for global optimization over continuous spaces," Journal of Global Optimization, vol. 11, no. 4, pp. 341359, 1997.

[31] K. Deb, S. Agrawal, A. Pratapand, and T. Meyarivan, "A fast elitist non-dominated sorting algorithm for multi-objective optimization: NSGA-II," in Parallel Problem Solving from NaturePPSN VI, pp. 849-858, Springer, Berlin, Germany, 2000.

[32] M. J. Reddy and D. N. Kumar, "An efficient multi-objective optimization algorithm based on swarm intelligence for engineering design," Engineering Optimization, vol. 39, no. 1, pp. 4968, 2007 .

[33] E. Zitzler, L. Thiele, M. Laumanns, C. M. Fonseca, and V. G. da Fonseca, "Performance assessment of multiobjective optimizers: an analysis and review," IEEE Transactions on Evolutionary Computation, vol. 7, no. 2, pp. 117-132, 2003. 

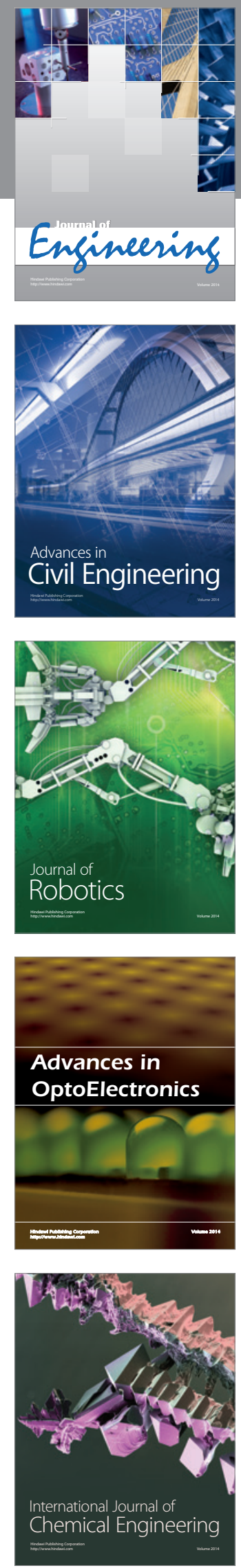

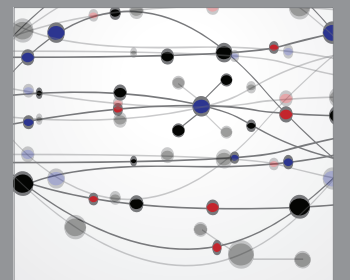

The Scientific World Journal
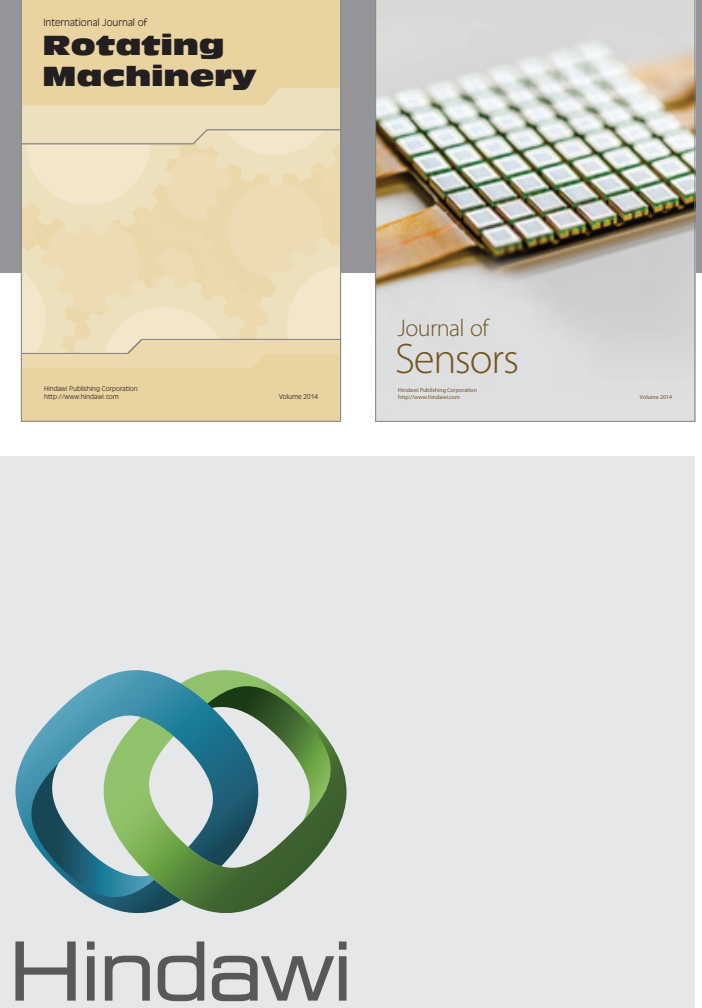

Submit your manuscripts at http://www.hindawi.com
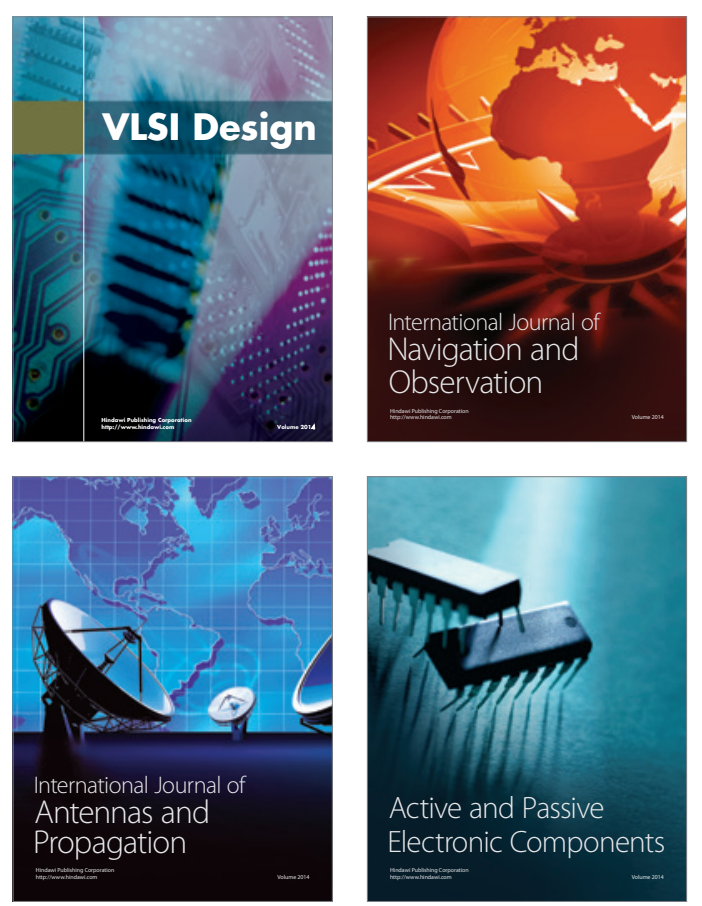
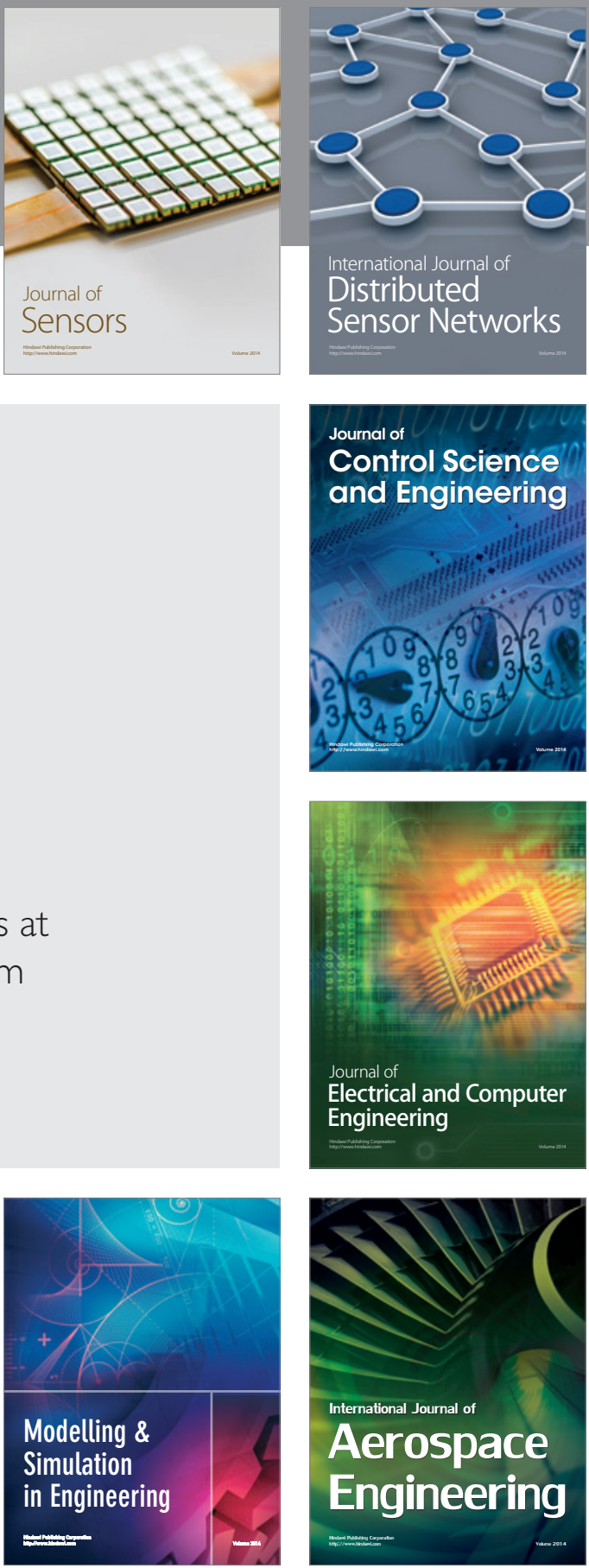

Journal of

Control Science

and Engineering
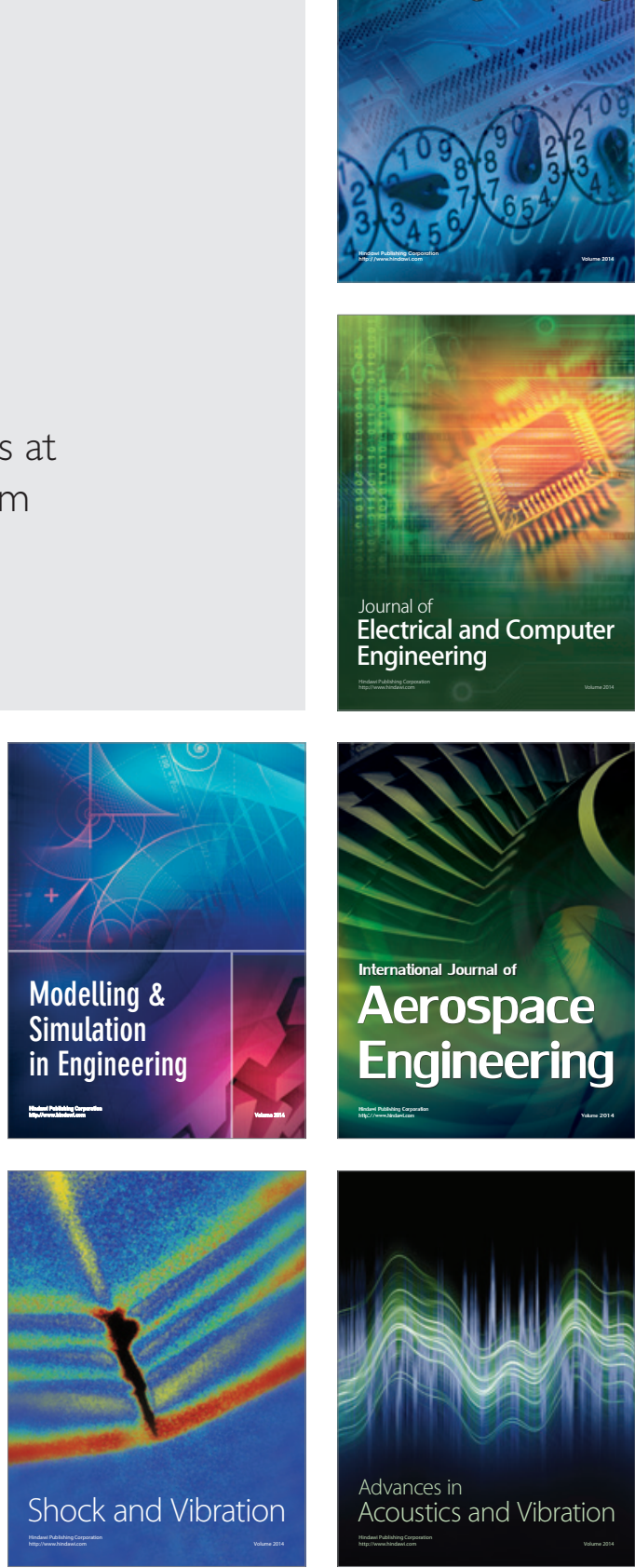